\title{
Examining the Relationships Among Item Recognition, Source Recognition, and Recall From an Individual Differences Perspective
}

\author{
Nash Unsworth and Gene A. Brewer \\ University of Georgia
}

\begin{abstract}
The authors of the current study examined the relationships among item-recognition, source-recognition, free recall, and other memory and cognitive ability tasks via an individual differences analysis. Two independent sources of variance contributed to item-recognition and source-recognition performance, and these two constructs related differentially to other memory and cognitive ability constructs. The results are in accordance with a dual-process theory of memory retrieval in which a familiarity process can support judgments of previous occurrence and a more strategic recollection process is needed for controlled search of long-term memory. Furthermore, the authors offer additional evidence in favor of a dual-process model by showing validity for these two unique sources of variance.
\end{abstract}

Keywords: dual process, item recognition, source recognition, individual differences

Episodic memory is critical for many tasks carried out everyday. In the laboratory, episodic memory can be measured with a variety of tasks including recall and recognition, as well as judgments of recency, frequency, and source. In recall tasks, participants must generate items, while in recognition tasks, participants are given an item and are asked to judge whether the item was presented during study (item recognition) or whether the item was presented at study and what the source of the item was (source recognition). Previous work has been devoted to examinations of the relations among these different tasks from experimental, neuropsychological, neuroimaging, and computational modeling perspectives. However, less work has been conducted on the relation among these various measures from an individual differences perspective. That is, few researchers have actually examined the correlations among these different tasks, and even fewer have attempted to model the relations among the tasks at a latent level. Our goal in the current study was to examine how recall, item recognition, and source recognition are related to one another and how they are related to other important cognitive constructs via latent variable analyses.

\section{Dual-Process Models of Recognition}

One influential class of models that nicely accounts for similarities and differences among recall, item-recognition, and sourcerecognition tasks are dual-process models of recognition. In these models, it is assumed that performance on recognition tasks is driven by two separate processes: a fast-acting, fairly automatic familiarity process and a slower, more controlled recollection

Nash Unsworth and Gene A. Brewer, Department of Psychology, University of Georgia.

Thanks to Greg Spillers for comments on an earlier version of this article.

Correspondence concerning this article should be addressed to Nash Unsworth, Department of Psychology, University of Georgia, Athens, GA 30602. E-mail: nunswor@uga.edu process (Jacoby, 1991; Yonelinas, 2002). In standard item recognition, participants can base their responding on either an automatic familiarity signal or on a controlled recollective-search process. ${ }^{1}$ In many cases, these two processes will lead to the same response. However, in situations requiring finer discriminations among items, the familiarity processes may lead to an incorrect response, and thus there will be a greater need for the controlled recollection process to recover information related to the target item. In source recognition, where the participants have to not only decide if the item was presented during study but also discriminate the source (male or female) of the item, recollection will primarily be needed to make an accurate judgment (Yonelinas, 1999). This is not to say that familiarity is absent in source recognition but that performance is mainly reliant on recollection (Hicks, Marsh, \& Ritschel, 2002). Item and source recognition should be related to the extent that both rely on recollection and, to a lesser extent, familiarity.

Recall is thought to rely on a controlled retrieval process similar to that found in recollection. According to dual-process models, item recognition and recall should be related to the extent that both tasks rely on recollection, although the relation might be weak depending on how much familiarity influences performance on item recognition. Likewise, because source recognition is thought to rely primarily on recollection, recall and source recognition should be highly related. Evidence consistent with dual-process models comes primarily from experimental and neuropsychological studies showing that estimates of familiarity and recollection can be dissociated (Parks \& Yonelinas, 2007). This work provides important evidence for the notion that at least two separate sources of variance account for performance in recognition tasks.

Dual-process models can be contrasted with single-process models that suggest that performance is not driven by separate mechanisms; rather performance on nearly all explicit episodic

\footnotetext{
${ }^{1}$ Note, we are agnostic as to whether recollection is better thought of as a continuous or a discrete process.
} 
memory tasks is driven by a single mechanism (memory strength). In these models, it is assumed that performance on many memory tasks relies on a single declarative memory system (Squire \& Zola, 1996) and that differences that arise are due to differences in memory strength. Performance on item recognition is generally better than performance on source-recognition and recall tasks because stronger memory traces are needed for accurate performance on source-recognition and recall tasks. Thus, single-process models assume that there are not separate sources of variance contributing to the different tasks; rather, there is a single, dominant source of variance that contributes to performance on all three types of tasks.

Despite the fact that dual-process and single-process models have been examined from various perspectives in a number of studies, examinations of these notions from an individual differences perspective have been conducted far less frequently. As noted by Underwood (1975), individual differences studies provide a litmus test for cognitive theories. If two constructs are thought to be related to one another, then this should be reflected in basic correlation analyses. Furthermore, if two constructs are thought to be independent, the correlation between them should be relatively small, and the two constructs should be differentially related to other constructs. This provides a means to test both convergent and discriminant validity of the constructs. To our knowledge, only three recent studies have been explicitly focused on dual-process models of recognition from an individual differences perspective (Oberauer, 2005; Quamme, Yonelinas, Widaman, Kroll, \& Sauvé, 2004; Yonelinas et al., 2007).

In the Oberauer (2005) study, participants performed either a local or global recognition task in which items were presented in separate frames onscreen. In global recognition, participants were given an item and were required to indicate whether the item belonged to the current set or not. Performance on this task could be based either on familiarity or recollection. In local recognition, participants were given an item presented in one of the frames and were asked to indicate if the location was correct. Oberauer (2005) argued that the local recognition task required mainly recollection because a judgment must be made not only concerning whether the item was a part of the current set but also concerning whether the item was in the correct frame. Oberauer (2005) performed several latent variable analyses to determine how familiarity and recollection would correlate with working memory (WM). Across all analyses, Oberauer found that recollection correlated with WM, but familiarity did not.

Similarly, Quamme et al. (2004) analyzed data on verbal fluency, free-recall, and item-recognition tasks for 54 patients with hypoxia. Quamme et al. tested a number of models including single-process, dual-process recall, and dual-process recognition models via structural equation modeling. For the dual-process recognition models, Quamme et al. allowed both the recognition and the free recall measures to load on a single latent factor (recollection), and the remaining recognition variance to load on another latent factor (familiarity). Quamme et al. (2004) found that this dual-process recognition model fit the data quite well and that the recollection factor was significantly correlated with verbal fluency, age, and coma duration.

In a recent follow-up, Yonelinas et al. (2007) performed a similar set of analyses on data from older adults. Yonelinas et al. found that hippocampal volume was uniquely related to a recol- lection factor composed of the recall and recognition tasks, whereas entorhinal volume was uniquely related to a familiarity factor composed of only the recognition tasks. Furthermore, these effects were significant even when the investigators controlled for age. Like the findings of the Oberauer (2005) and Quamme et al. (2004) studies, these results suggest that there are two sources of variance in recognition tasks; with one source (recollection) showing greater correlations with other memory measures than the other source (familiarity), and these two sources of variance seem to be selectively related to the hippocampus (recollection) or the entorhinal cortex (familiarity).

\section{Current Study}

The Oberauer (2005), Quamme et al. (2004), and Yonelinas et al. (2007) studies provide initial support for dual-process theories from an individual differences perspective. However, there are a few limitations of these studies that could limit their generalizabilty. First, the Oberauer (2005) study specifically examined short-term recognition tasks in which only a few items were presented, and then participants were tested immediately. It is possible that the use of short-term recognition tasks biased the use of more recollection-like processes, whereby responses were based on traces that were still active in WM, and thus the models examined by Oberauer (2005) might not generalize to traditional long-term recognition tasks. The Quamme et al. (2004) study does not have this issue because recognition was tested on a larger set of items after a significant delay. However, the fact that participants in Quamme et al. (2004) were all hypoxic patients does limit the applicability of these results to healthy individuals. Clearly, the use of these patients is a strength of the Quamme et al. (2004) study in that they were primarily interested in examining dualprocess recognition models from both an individual differences and neuropsychological perspective. However, given the patients' deficits in basic memory tasks, it is not clear whether similar models will account for healthy young adults' performance. Additionally, although their sample size was large for a neuropsychological study, the use of only 54 participants is quite small for typical structural equation modeling studies in which relatively complex models are tested. The Yonelinas et al. (2007) study used a much larger number of participants $(N=157)$, and found results similar to those of the Quamme et al. (2004) study, yet all of these participants were older adults; thus, it is not clear whether these results will generalize to other populations. Finally, although Oberauer (2005), Quamme et al. (2004), and Yonelinas et al. (2007) examined the differential relation of the recollection and familiarity factors to other memory factors, they did not examine a broader range of memory and cognitive ability tasks to determine the convergent and discriminant validity of the two factors.

In the current study, we sought to examine single- and dualprocess models of recognition memory from an individual differences perspective with a large sample of healthy young adults on measures of item recognition, source recognition, and recall, as well as other memory and cognitive ability measures. In addition to the item-recognition, source-recognition and recall tasks, measures of WM, judgments of recency (JOR), and fluid abilities (gF) were administered. WM measures were administered because previous work has suggested that WM should be related to recollection and not familiarity (Oberauer, 2005), given that WM is needed 
to engage in a controlled search of memory similar to recollection (Unsworth \& Engle, 2007). JOR measures were given because previous work has suggested that when two items are presented in close temporal proximity, recollection will be needed to provide an accurate JOR (Konishi et al., 2002). Finally, gF measures were given to provide a broad-based cognitive abilities factor to determine how recollection and familiarity are differentially related to higher order cognition.

\section{Method}

\section{Participants}

A total of 172 participants were recruited from the subject pool at the University of Georgia. Participants were between the ages of 18 and 35 and received course credit for their participation. Each participant was tested individually in two laboratory sessions lasting approximately $2 \mathrm{hr}$ each.

\section{Materials and Procedure}

After signing informed consent, all participants completed the following tasks in order: operation span, symmetry span, reading span, delayed free recall with category switches, item recognition with words, item recognition with pictures, Raven's Advanced Progressive Matrices, verbal analogies, number series, cued recall with words, JOR with words, and JOR with pictures in Session 1. In Session 2, all participants completed delayed free recall, cued recall with numbers, gender-source recognition, and picture-source recognition.

For the tasks in which words were used as stimuli, we selected three- to six-letter medium-frequency nouns (average frequency 62.47) from the Toronto Word Pool (Friendly, Franklin, Hoffman, \& Rubin, 1982). Pictures in the item-recognition, sourcerecognition, and JOR tasks were taken from Snodgrass and Vanderwart (1980). For the recall and recognition tasks, all items were initially randomized, and then all participants received the same fixed order of trials. For all of the recall tasks, each response was hand scored to ensure that misspelled items were scored as correct.

\section{Tasks}

Operation span. Participants solved a series of math operations while trying to remember a set of unrelated letters. Participants were required to solve a math operation, and after solving the operation, they were presented with a letter for $1 \mathrm{~s}$. Immediately after the letter was presented, the next operation was presented. At recall, participants were asked to recall letters from the current set in the correct order by clicking on the appropriate letters. For all of the span measures, items were scored if the item was correct and in the correct position. The score was the number of correct items in the correct position.

Symmetry span. Participants recalled sequences of red squares within a matrix while performing a symmetry-judgment task. In the symmetry-judgment task, participants were shown an $8 \times 8$ matrix with some squares filled in black. Participants decided whether the design was symmetrical about its vertical axis. The pattern was symmetrical half of the time. Immediately after determining whether the pattern was symmetrical, participants were presented with a $4 \times 4$ matrix with one of the cells filled in red for $650 \mathrm{~ms}$. At recall, participants recalled the sequence of red-square locations in the preceding displays in the order they appeared by clicking on the cells of an empty matrix. We used the same scoring procedure as we used in the operation span.

Reading span. While trying to remember a set of unrelated letters, participants were required to read a sentence and to indicate whether it made sense or not. Half of the sentences made sense, while the other half did not. (We made nonsense sentences by simply changing one word in an otherwise normal sentence.) After participants gave their response, they were presented with a letter for $1 \mathrm{~s}$. At recall, participants were asked to recall letters from the current set in the correct order by clicking on the appropriate letters. We used the same scoring procedure as we used in the operation span.

Delayed free recall. Participants recalled six lists of 10 words each. Words were presented alone for $1 \mathrm{~s}$ each. After list presentation, participants performed a distractor task for $16 \mathrm{~s}$ in which a three-digit number appeared for $2 \mathrm{~s}$, and participants wrote the digits in ascending order. After the distractor task, participants typed as many words as they could remember from the list, one at a time, in any order they wished. Participants had $45 \mathrm{~s}$ for recall. ${ }^{2}$ A participant's score was the total number of items recalled correctly.

Delayed free recall with category switches. Participants recalled six lists of 10 words each, broken down into two blocks. All words in each block came from the same semantic category. Words were presented alone for $1 \mathrm{~s}$ each. Following presentation of the last word in a list, participants were required to count backwards by three's from a three-digit number shown onscreen for $15 \mathrm{~s}$ and to write the numbers down. After the distractor task, participants typed as many words as they could remember from the list, one at a time, in any order they wished. Participants had $45 \mathrm{~s}$ for recall. A participant's score was the total number of items recalled correctly.

Cued-recall words. Participants were given three lists of 10 word pairs each. The word pairs were presented vertically for $2 \mathrm{~s}$ each. Participants were told that the cue would always be the word on the top and the target would always be the word on the bottom. After the presentation of the last word, participants saw the cue word and "???" in place of the target word. Participants recalled the word from the list that matched the cue. Cues were randomly mixed so that the corresponding target words were not recalled in the same order as they were presented. Participants had $5 \mathrm{~s}$ to type in the corresponding word. A participant's score was proportion of correctly recalled words.

Cued-recall numbers. This task was exactly the same as the cued-recall task with words but instead of word-word pairs, a random three-digit number served as the cue that was paired with

\footnotetext{
${ }^{2}$ Note, prior research has suggested that $45 \mathrm{~s}$ is ample time for free recall with the given list lengths (Unsworth, 2008). Furthermore, note that prior to the recall tasks, all participants completed a brief typing exercise (typing the words for numerals from one to ten) to estimate their typing efficiency. Typing speed was not related to any of the variables in the current study (all $r \mathrm{~s}<.09$, all $p \mathrm{~s}>$.16). It is important to note that partialling out typing speed did not change any of the results. Thus, individual differences in typing speed did not account for any of the results of the current study.
} 
the target word. A participant's score was proportion of correctly recalled words.

Item-recognition words. Participants were presented with 30 words onscreen at encoding. Words were presented individually for $2 \mathrm{~s}$ each. At test, participants were presented with one word in the center of the screen and were asked to indicate whether the word was old or new via key press. Participants were tested on 30 old and 30 new words. Participants had $5 \mathrm{~s}$ to press the appropriate key to enter their response. A participant's score was proportion correct.

Item-recognition pictures. Participants were presented with 30 pictures onscreen at encoding. Each picture was presented individually for $3 \mathrm{~s}$ each. At test, participants were presented with one picture in the center of the screen and were asked to indicate whether the picture was old or new via key press. Participants were tested on 30 old and 30 new pictures. Participants had $5 \mathrm{~s}$ to press the appropriate key to enter their response. A participant's score was proportion correct.

Gender-source recognition. Participants heard words (30 total words) spoken by either a male or a female voice. Participants were explicitly instructed to pay attention to both the word and the voice in which the word was spoken. At test, participants were presented with 30 old and 30 new words and were required to indicate via key press whether the word was new or old and, if the word was old, whether the voice in which the word had been spoken had been male or female. Participants had $5 \mathrm{~s}$ to press the appropriate key to enter their response. Conditionalized sourceidentification measures were computed for both sources. For example, all of the male items called male from all male items attributed to the study list (i.e., those labeled male or female) represented a participant's male conditionalized sourceidentification measure. A participant's score was the average of these two conditionalized source-identification measures.

Picture-source recognition. Participants were presented with a picture (30 total pictures) in one of four different quadrants onscreen for $1 \mathrm{~s}$. Participants were explicitly instructed to pay attention to both the picture as well as the quadrant in which it was located. At test, participants were presented with 30 old and 30 new pictures in the center of the screen. Participants were asked to indicate via key press whether the picture was new or old and, if the picture was old, the quadrant in which it was presented. Participants had $5 \mathrm{~s}$ to press the appropriate key to enter their response. The same scoring procedure as gender-sourcerecognition was used.

JOR words. Participants were presented with three lists of 10 words each. Each word was presented individually onscreen for $2 \mathrm{~s}$. At test, participants were given two words from the current list and were required to indicate via key press which word had been presented more recently than the other. Participants had $5 \mathrm{~s}$ to press the appropriate key to enter their response. A participant's score was the proportion correct.

JOR pictures. This task was exactly the same as that for JOR words, but instead of words, pictures were presented. A participant's score was the proportion correct.

Raven's advanced progressive matrices. Participants received 36 items presented in ascending order of difficulty. Each item consisted of a display of $3 \times 3$ matrices of geometric patterns with the bottom right pattern missing. Participants selected the correct answer from eight alternatives. Participants had $10 \mathrm{~min}$ to com- plete the 18 odd-numbered items. A participant's score was the total number of correct solutions.

Verbal analogies. Participants read an incomplete analogy and were required to select the one word out of five possible words that best completed the analogy. Participants had 5 min to complete 18 test items. A participant's score was the total number of items solved correctly.

Number series. Participants saw a series of numbers and were required to determine what the next number in the series should be. That is, the series followed some unstated rule or pattern that participants attempted to figure out in order to determine what the next number in the series should be. Participants selected their answer out of five possible numbers that were presented. Participants had $4.5 \mathrm{~min}$ to complete 15 test items. A participant's score was the total number of items solved correctly.

\section{Results}

Descriptive statistics and correlations for the measures are shown in Table 1 . All measures had generally acceptable values of internal consistency, and most of the measures were approximately normally distributed with values of skewness and kurtosis under the generally accepted values.

Next, we tested several latent variable models via confirmatory factor analysis to examine the extent to which item recognition, source recognition, and recall depend on the same or different cognitive processes. In Model 1, the item-recognition, sourcerecognition, and recall tasks all loaded on the same memory factor representing a single-process model. This factor was allowed to correlate with separate WM, JOR, and gF factors. Thus, in this model, all of the memory factors loaded onto a single unitary factor. In Model 2, we examined the question of whether there were differences between recall and recognition by having the four recall tasks load on one factor and the four recognition (both item and source) load onto a separate factor. That is, with this model, we tested the extent to which a similar process drives performance in recognition tasks (i.e., single-process recognition), but this is different from recall. The separate recognition and recall factors were allowed to correlate with one another and with the other three factors. Finally, the extent to which item recognition, source recognition, and recall represent distinct cognitive constructs was examined in Model 3. ${ }^{3}$ That is, with this model, we tested the extent to which slightly different processes are needed in the three classes of tasks. As noted earlier, according to dual-process models of recognition, source recognition should be related to both item recognition and recall but should be more highly related to recall to the extent that both are reliant on recollection. In this model, the two item-recognition tasks loaded on one factor, the two sourcerecognition tasks loaded on a second factor, and the four recall factors loaded on a third factor. These factors were allowed to correlate with one another and with the other three factors. Thus, these three models tested the extent to which the three classes of tasks were best represented by a single factor, two factors (one for

\footnotetext{
${ }^{3}$ Note, although some of these model configurations may seem intuitively more plausible than others, it is important to test the extent to which the models differentially account for the data given possible theoretical and methodological differences.
} 
Table 1

Means, Standard Deviations, Estimates of Reliability, and Correlations for All Measures

\begin{tabular}{|c|c|c|c|c|c|c|c|c|c|c|c|c|c|c|c|c|}
\hline Measure & 1 & 2 & 3 & 4 & 5 & 6 & 7 & 8 & 9 & 10 & 11 & 12 & 13 & 14 & 15 & 16 \\
\hline 1. ItemW & - & & & & & & & & & & & & & & & \\
\hline 2. ItemP & .57 & - & & & & & & & & & & & & & & \\
\hline 3. GenSour & .12 & .01 & - & & & & & & & & & & & & & \\
\hline 4. PicSour & .30 & .19 & .23 & - & & & & & & & & & & & & \\
\hline 5. DFR & .27 & .19 & .18 & .32 & - & & & & & & & & & & & \\
\hline 6. DFRC & .34 & .17 & .18 & .18 & .26 & - & & & & & & & & & & \\
\hline 7. CRW & .23 & .12 & .17 & .26 & .37 & .27 & - & & & & & & & & & \\
\hline 8. CRN & .23 & .18 & .19 & .24 & .45 & .19 & .33 & - & & & & & & & & \\
\hline 9. Ospan & .23 & .23 & .12 & .20 & .09 & .23 & .17 & .17 & - & & & & & & & \\
\hline 10. SymSpan & .12 & .09 & .20 & .13 & .06 & .19 & .02 & .13 & .48 & - & & & & & & \\
\hline 11. Rspan & .22 & .09 & .12 & .10 & .09 & .18 & .18 & .12 & .53 & .38 & - & & & & & \\
\hline 12. JORW & .13 & .02 & .13 & .08 & .10 & .11 & .12 & .12 & -.01 & .08 & .01 & - & & & & \\
\hline 13. JORP & .14 & .08 & .15 & .14 & .15 & .08 & .14 & .16 & .05 & .09 & -.01 & .62 & - & & & \\
\hline 14. Raven & .20 & .22 & .14 & .18 & .21 & .18 & .30 & .23 & .23 & .33 & .22 & .17 & .16 & - & & \\
\hline 15. NS & .21 & .16 & .02 & .06 & .05 & .15 & .12 & .09 & .25 & .29 & .22 & .05 & .02 & .20 & - & \\
\hline 16. Ang & .28 & .26 & .17 & .11 & .12 & .19 & .28 & .07 & .36 & .23 & .27 & .10 & .13 & .43 & .20 & - \\
\hline$M$ & 0.83 & 0.96 & 0.71 & 0.78 & 34.06 & 36.04 & 0.24 & 0.13 & 60.69 & 30.52 & 58.06 & 0.57 & 0.72 & 10.65 & 9.81 & 11.71 \\
\hline$S D$ & 0.09 & 0.05 & 0.14 & 0.16 & 8.96 & 6.15 & 0.22 & 0.11 & 11.86 & 7.20 & 13.69 & 0.26 & 0.28 & 2.28 & 2.57 & 2.77 \\
\hline Rel & 0.67 & 0.65 & 0.62 & 0.72 & 0.79 & 0.71 & 0.83 & 0.68 & 0.80 & 0.78 & 0.82 & 0.73 & 0.86 & 0.72 & 0.80 & 0.69 \\
\hline Skew & -1.14 & -5.21 & -0.11 & -1.12 & 0.22 & 0.03 & 0.87 & 0.97 & -1.91 & -0.82 & -1.41 & -0.38 & -1.11 & -0.11 & -0.17 & -0.51 \\
\hline Kurtosis & 3.34 & 41.54 & -0.65 & 1.52 & -0.27 & 1.32 & -0.35 & 0.37 & 0.64 & 1.03 & 2.03 & -0.61 & 0.37 & -0.21 & -0.42 & -0.12 \\
\hline
\end{tabular}

Note. $\quad$ ItemW = item-recognition words; ItemP = item-recognition pictures; GenSour = gender-source recognition; PicSour = picture-source recognition; $\mathrm{DFR}=$ delayed free recall; DFRC $=$ delayed free recall categorically related words; $\mathrm{CRW}=$ cued-recall words; $\mathrm{CRN}=$ cued-recall numbers; Ospan $=$ operation span; SymSpan = symmetry span; Rspan = reading span; JORW = judgments of recency words; JORP = judgment of recency pictures; Raven = Raven's Advanced Progressive Matrices; NS = number series; Ang = verbal analogies; Rel = reliability.

recognition and one for recall), or three separate yet correlated factors. Shown in Table 2 are the fit statistics for each of the models. As can be seen, although all of the models fit relatively well, Model 3 fit the data the best, both $\Delta \chi^{2} \mathrm{~s}>28, p \mathrm{~s}<.01$, and had the lowest Akaike's information criterion value.

The estimates for Model 3 are shown in Figure 1. As can be seen, item recognition was moderately correlated with both source recognition and recall. The source and recall factors were strongly correlated. However, inspection of the correlations suggested some differences. For instance, both the recall and source factors were moderately related to the WM, JOR, and gF factors, whereas the item-recognition factor was moderately related to the $\mathrm{WM}$ and $\mathrm{gF}$ factors but was not significantly related to the JOR factor. This suggests that there were commonalities and differences between the factors, with the source and recall factors being highly related to one another and both being related to the item-recognition factor.

Because of the high correlation between the recollection and recall factors, we more formally tested the dual-process model with the last model. In this model (Model 4), a recollection factor was specified on the basis of shared variance across the itemrecognition, source-recognition, and recall tasks (Quamme et al., 2004; Yonelinas et al., 2007). The familiarity factor was specified as the variance associated with item recognition that was independent of the variance shared between the item-recognition and source-recognition and recall tasks. The correlation between the two factors was set to zero. In this way, it was possible to extract separate sources of variance from the tasks and examine the extent to which these separate sources of variance were related to the other factors. ${ }^{4}$ As shown in Table 2, the fit of the model was good.
As shown in Figure 2, the loadings of the manifest variables onto both the familiarity and recollection factors were significant. Furthermore, the recollection factor was moderately correlated with the recall, WM, JOR, and $\mathrm{gF}$ factors. The familiarity factor was only significantly related to the $\mathrm{gF}$ factor. Consistent with previous work (Oberauer, 2005; Quamme et al., 2004), recollection was related to a number of cognitive constructs that are thought to rely, at least partially, on recollection-like processes, but familiarity was not related to these constructs. This provides important evidence for the notion that at least two sources of variance underlie these tasks, and these two sources of variance are differentially related to other cognitive constructs.

\section{Discussion}

In the current study, we examined the relations among item recognition, source recognition, and recall from an individual differences perspective. We found that a three factor model in which item recognition, source recognition, and recall were treated as separate factors fit the data significantly better than either a single-factor (single-process model) or a two-factor (singleprocess recognition and separate-recall model) model. Furthermore, a more formal dual-process model in which the variance shared by item recognition, source recognition, and recall formed

\footnotetext{
${ }^{4}$ Note, we also tried a model in which the source-recognition tasks loaded on the familiarity factor; however, neither loading was significant. Thus, only the model with item-recognition tasks loading on the familiarity factor is shown.
} 
Table 2

Fit Indices For All Models

\begin{tabular}{lcccccrrr}
\hline \multicolumn{1}{c}{ Model no. and name } & $\chi^{2}$ & $d f$ & $\chi^{2} / d f$ & RMSEA & NNFI & CFI & SRMR & AIC \\
\hline Model 1: Single process & 134.10 & 98 & 1.37 & .05 & 0.95 & 0.96 & .06 \\
Model 2: Recall-recognition & 110.74 & 94 & 1.18 & .03 & 0.98 & 0.98 & .06 \\
Model 3: Three memory factors & 81.74 & 89 & 0.92 & .00 & 1.00 & 1.00 & .05 & 194.74 \\
Model 4: Recollection-familiarity & 84.54 & 93 & 0.91 & .00 & 1.00 & 1.00 & .05 & 170.54 \\
\hline
\end{tabular}

Note. $\quad$ RMSEA $=$ root-mean-square error of approximation; NNFI $=$ nonormed fit index; CFI $=$ comparative fit index; SRMR $=$ standardized root-mean-square residual; AIC = Akaike's information criterion

one factor (recollection) and the independent item recognition variance formed another factor (familiarity) showed that recollection was related to $\mathrm{WM}$, JORs, and $\mathrm{gF}$, but familiarity was only related to $\mathrm{gF}$.

These results are consistent with dual-process models of recognition in which both recollection and familiarity are important for performance. Specifically, the results suggest that at least two sources of variance contribute to performance on recognition tasks, and these two sources of variance are differentially related to other cognitive abilities. Thus, the results provide convergent and discriminant validity for the notion of recollection and familiarity and for dual-process models of recognition more generally. In fact, it is

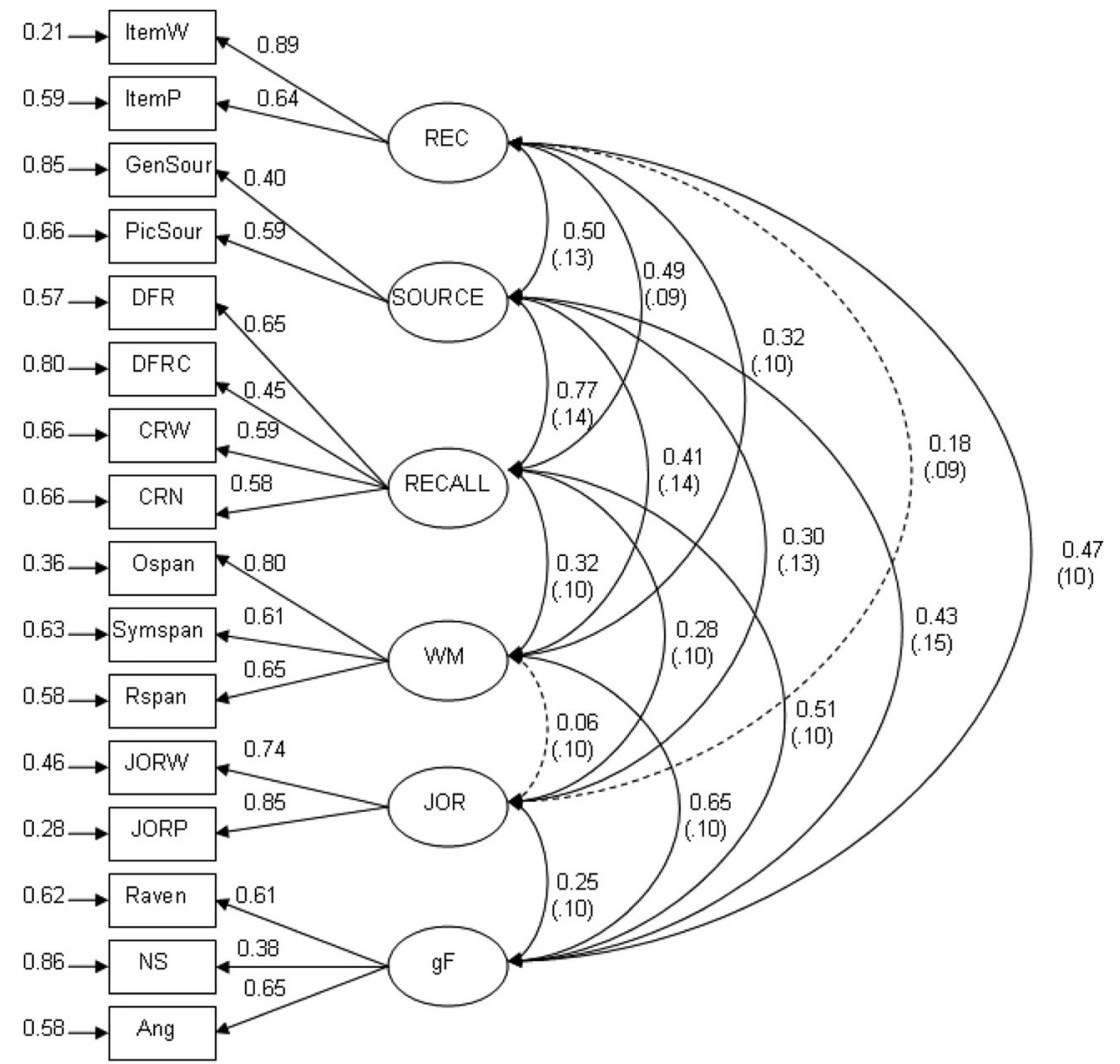

Figure 1. Confirmatory factor analysis for item recognition (REC), source recognition (SOURCE), recall, working memory (WM), judgments of recency (JOR), and general fluid intelligence ( $\mathrm{gF}$ ). Paths connecting latent variables (circles) to each other represent the correlations between the constructs, the numbers from the latent variables to the manifest variables (squares) represent the loadings of each task onto the latent variable, and numbers appearing next to each manifest variable represent error variance associated with each task. Solid lines are significant at the $p<.05$ level, and dotted lines are not significant at the $p<.05$ level. Numbers in parentheses are standard errors. Standard errors for the factor loadings were all $<.14$. ItemW $=$ item-recognition words; ItemP $=$ item-recognition pictures; GenSour $=$ gender-source recognition; PicSour $=$ picture-source recognition; DFR = delayed free recall; DFRC = delayed free recall with category switches; CRW = cued-recall words; $\mathrm{CRN}=$ cued-recall numbers; Ospan $=$ operation span; Symspan $=$ Symmetry span; Rspan $=$ reading span; JORW = judgment of recency words; JORP = judgment of recency pictures; Raven = Raven's Advanced Progressive Matrices; NS $=$ number series; $\mathrm{ANG}=$ analogies. 


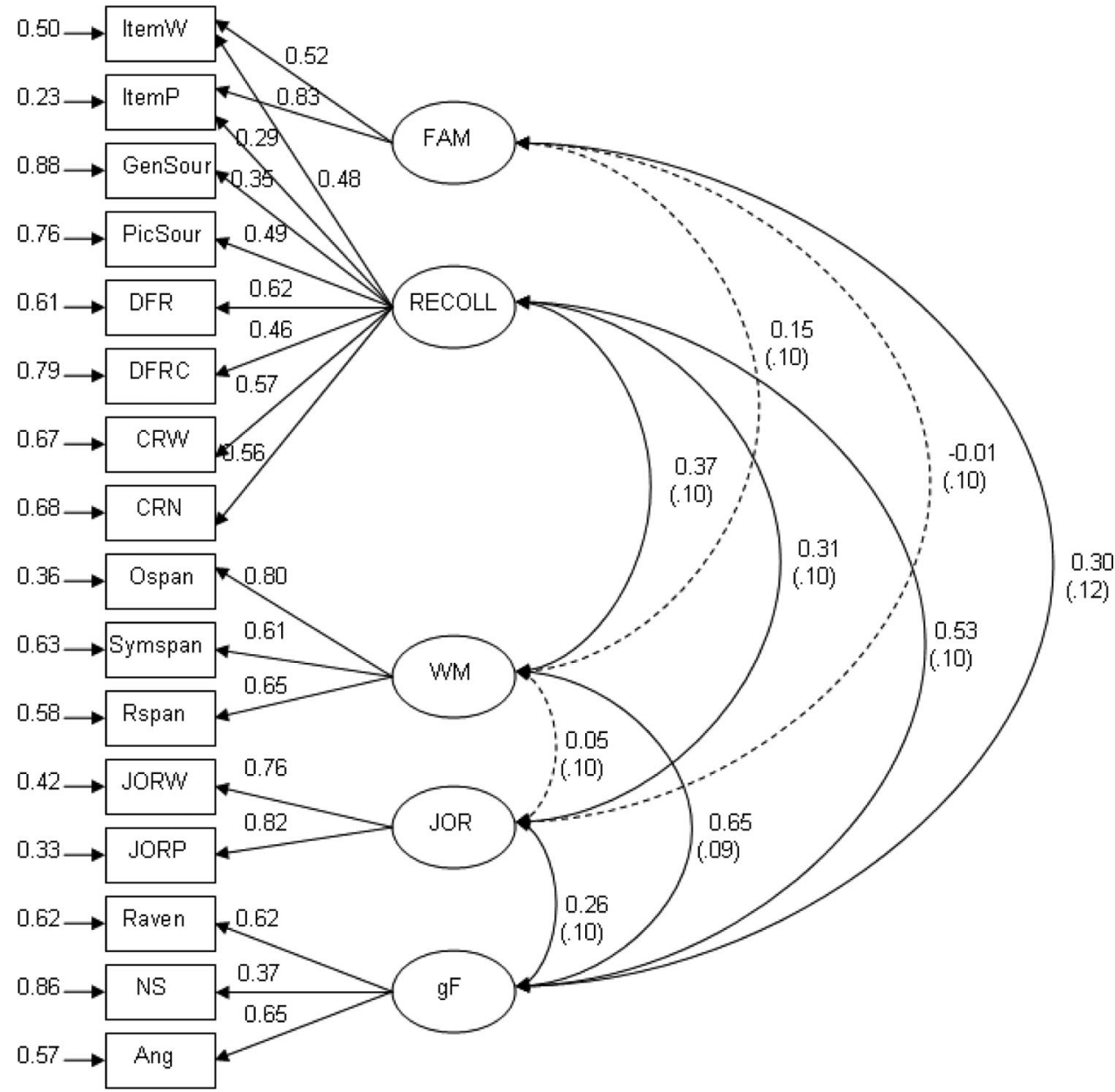

Figure 2. Confirmatory factor analysis for familiarity (FAM), recollection (RECOLL), working memory (WM), judgments of recency (JOR), and general fluid intelligence ( $\mathrm{gF}$ ). Paths connecting latent variables (circles) to each other represent the correlations between the constructs, the numbers from the latent variables to the manifest variables (squares) represent the loadings of each task onto the latent variable, and numbers appearing next to each manifest variable represent error variance associated with each task. Solid lines are significant at the $p<.05$ level, and dotted lines are not significant at the $p<.05$ level. Numbers in parentheses are standard errors. Standard errors for the factor loadings were all $<.14$. ItemW $=$ item-recognition words; ItemP $=$ item-recognition pictures; GenSour $=$ gender-source recognition; PicSour $=$ picture-source recognition; DFR $=$ delayed free recall; DFRC $=$ delayed free recall with category switches; CRW $=$ cued-recall words; $\mathrm{CRN}=$ cued-recall numbers; Ospan $=$ operation span; Symspan $=$ Symmetry span; Rspan $=$ reading span; JORW $=$ judgment of recency words; JORP $=$ judgment of recency pictures; Raven $=$ Raven's Advanced Progressive Matrices; NS = number series; $\mathrm{ANG}=$ analogies.

not clear how single-process models would account for the current results in that by their very nature they suggest that only a single process determines performance. The finding that single-process covariation models did not fit the data as well as dual-process models and the finding that there are two independent sources of variance that determine performance provide evidence for dualprocess models over single-process models. The current results extend prior work on dual-process models because the results rely on an individual differences framework (Oberauer, 2005; Quamme et al., 2004; Yonelinas et al., 2007) by showing that essentially the same dual-process model is found with very different samples (young adults, hypoxic patients, older adults) as well as with different measures (item recognition, source recognition, free recall, cued recall) over both the short and the long term.

Other dual-component models, such as generation and recognition or strength-plus-decision processes, do not clearly map onto the parameterization found in Model 4. Specifically, although Model 4 depicts two independent sources of variance as being important, neither of these sources map readily onto generation and recognition or strength-plus-decision processes. Rather, the particular configuration of Model 4 corresponds with an abundance of evidence supporting a distinction between recollection and familiarity. Thus, it is not the case that any dual-component 
model will account for the data but rather that this particular dual-process model does.

Overall, the results are in accordance with a dual-process theory of memory retrieval in which a familiarity process can support judgments of previous occurrence and a more strategic recollection process is needed for controlled search of long-term memory. Previous theorizing has suggested that while performing recognition tasks, one can rely on perceptual or conceptual fluency processes (familiarity), while at other times more controlled-strategic retrieval will be needed (recollection; Jacoby, 1991). For the tasks administered in the current study, participants tend to rely more on controlled retrieval, given the need to make fine grained discriminations. These controlled-strategic retrieval processes are also likely needed on measures of WM, JORs, and $\mathrm{gF}$.

Specifically, prior work has suggested that WM (Unsworth \& Engle, 2007) is reliant on controlled-strategic search processes like recollection in which information that has been displaced from the focus of attention needs to be retrieved from long-term memory via a controlled search. Similarly, Konishi et al. (2002) have suggested that recollection will be needed for fine-grained temporal discrimination in JOR tasks when two items are presented in close temporal proximity and a simple assessment of prior occurrence will not lead to the correct response. Thus, the common variance associated with the recollection factor and the other cognitive ability factors likely represents the need to engage in controlled retrieval (Dobbins, Foley, Schacter, \& Wagner, 2002; Jacoby, 1991), which is reliant on the hippocampus and prefrontal cortex. Individuals with greater controlled retrieval abilities will demonstrate better performance on those tasks that require a search of memory than individuals with less controlled retrieval abilities. When performance is driven by perceptual or conceptual fluency, however, individual differences in controlled retrieval will not matter, leading to weak or nonexistent correlations with measures such as WM. This variance will be related to measures that rely on perceptual fluency processes in which the ability to recognize common perceptual patterns across trials is important (as is the case with many measures of $\mathrm{gF}$ in which participants are required to recognize patterns). Thus, both sources of variance (recollection and familiarity) are needed to account for performance on recognition tasks and to account for their relation with other cognitive ability constructs.

\section{References}

Dobbins, I. G., Foley, H., Schacter, D. L., \& Wagner, A. D. (2002). Executive control during episodic retrieval: Multiple prefrontal processes subserve source memory. Neuron, 35, 989-996.

Friendly, M., Franklin, P. E., Hoffman, D., \& Rubin, D. C. (1982). The Toronto Word Pool: Norms for imagery, concreteness, orthographic variables, and grammatical usage for 1,080 words. Behavior Research Methods and Instruments, 14, 375-399.

Hicks, J. L., Marsh, R. L., \& Ritschel, L. (2002). The role of recollection and partial information in source monitoring. Journal of Experimental Psychology: Learning, Memory, and Cognition, 28, 503-508.

Jacoby, L. L. (1991). A process-dissociation framework: Separating automatic from intentional uses of memory. Journal of Memory and Language, 30, 513-541.

Konishi, S., Uchida, I., Okuaki, T., Machida, T., Shirouzu, I., \& Miyashita, Y. (2002). Neural correlates of recency judgment. The Journal of Neuroscience, 22, 9549-9555.

Oberauer, K. (2005). Binding and inhibition in working memory: Individual and age differences in short-term recognition. Journal of Experimental Psychology: General, 134, 368-387.

Parks, C. M., \& Yonelinas, A. P. (2007). Moving beyond pure signaldetection models: Comment on Wixted. Psychological Review, 114, $188-202$.

Quamme, J. R., Yonelinas, A. P., Widaman, K. F., Kroll, N. E. A., \& Sauvé, M. J. (2004). Recall and recognition in mild hypoxia: Using covariance structural modeling to test competing theories of explicit memory. Neuropsychologia, 42, 672-691.

Snodgrass, J. G., \& Vanderwart, M. (1980). A standardized set of 260 pictures: Norms for name agreement, image agreement, familiarity, and visual complexity. Journal of Experimental Psychology: Human Learning \& Memory, 6, 174-215.

Squire, L. R., \& Zola, S. M. (1996). The structure and function of declarative and nondeclarative memory systems. Proceedings of the National Academy of Sciences of the United States of America, 93, 13515-13522.

Underwood, B. J. (1975). Individual differences as a crucible in theory construction. American Psychologist, 30, 128-134.

Unsworth, N. (2008). Exploring the retrieval dynamics of delayed and final free recall: Further evidence for temporal-contextual search. Journal of Memory and Language, 59, 223-236.

Unsworth, N., \& Engle, R. W. (2007). The nature of individual differences in working memory capacity: Active maintenance in primary memory and controlled search from secondary memory. Psychological Review, 114, 104-132.

Yonelinas, A. P. (1999). The contribution of recollection and familiarity to recognition and source memory: An analysis of receiver operating characteristics and a formal model. Journal of Experimental Psychology: Learning, Memory, and Cognition, 25, 1415-1434.

Yonelinas, A. P. (2002). The nature of recollection and familiarity: A review of 30 years of research. Journal of Memory and Language, 46, $441-517$.

Yonelinas, A. P., Widaman, K., Mungas, D., Reed, B., Weiner, M. W., \& Chui, H. C. (2007). Memory in the aging brain: Doubly dissociating the contribution of the hippocampus and entorhinal cortex. Hippocampus, 17, 1134-1140.

Received December 16, 2008

Revision received July 14, 2009

Accepted July 20, 2009 